\title{
Temporal Wilson loop in the Hamiltonian approach in Coulomb gauge
}

\author{
Markus Quandt* \\ Universität Tübingen \\ E-mail: markus . quandteuni-tuebingen. de \\ H. Reinhardt \\ Universität Tübingen \\ E-mail: hugo.reinhardteuni-tuebingen. de \\ G. Burgio \\ Universität Tübingen \\ E-mail: giuseppe.burgio@uni-tuebingen.de
}

\begin{abstract}
We investigate the temporal Wilson loop using the Hamiltonian approach to Yang-Mills theory. In simple cases such as the Abelian theory or the non-Abelian theory in $(1+1)$ dimensions, the known results can be derived using unitary transformations to take care of time evolution. Alternatively, the exact solution can also be found in Coulomb gauge using the exact ground state wave functional which is known explicitly in these simple cases. The Coulomb gauge technique can also be applied to the more realistic case of Yang-Mills theory in $(3+1)$ dimensions, where one has to rely on the approximate vacuum wave functional obtained e.g. in recent variational approaches. We use this formulation to compute the temporal Wilson loop in $(3+1)$ dimensional Yang-Mills theory, and find that the Wilson and Coulomb string tension agree within this approximation scheme. Possible improvements of these findings are briefly discussed.
\end{abstract}

Xth Quark Confinement and the Hadron Spectrum

8-12 October 2012

TUM Campus Garching, Munich, Germany

${ }^{*}$ Speaker. 


\section{Introduction}

Recent efforts to study Yang-Mills (YM) theory are largely based on Coulomb gauge, because the Gribov-Zwanziger picture of confinement $[1,2]$ becomes particularly transparent in this framework: the only constraint on the physical states in a Hamiltonian approach based on Coulomb gauge is Gau $\beta$ ' law, which in turn can be resolved exactly so that any normalizable wave functional can be used as a physical state.

Such a formulation naturally lends itself to variational methods, which are usually based on (modified) Gaussian Ansätze for the vacuum wave functional, see e.g. [3, 4] and references therein. The solutions agree with the Gribov-Zwanziger scenario and, when combined with the horizon condition, lead to a coherent picture of strongly coupled YM theory, which is mainly dominated by a diverging gluon energy in the infrared, a linearly rising Coulomb potential between static quarks, and a strongly enhanced Faddeev-Popov ghost propagator in the infrared. The latter condition implies that the YM vacuum behaves as a perfect colour dia-electric medium [5], i.e. a dual superconductor. All these findings agree qualitatively with the results from recent lattice simulations [6], although there are still some discrepancies in the quantitative details, in particular for the ghost sector [7].

However, this simple picture is subject to the caveat that the Coulomb potential is only an upper bound for the true potential between heavy quarks [8]. For a complete description, one has to address the physical potential from large Wilson loops directly. In the continuum, this is complicated by path ordering and large self-energy contributions which obscure the extraction of the true potential from large Wilson loops [9].

In this talk, I report on a recent approach [10] to compute the Wilson potential in the Hamiltonian approach in Coulomb gauge. A careful analysis of Gauß' law and the physical degrees of freedom allows for an elegant way to isolate and remove the self-energy contributions to the Wilson loop, while unitary transformations are applied to take care of time evolution. As specific examples, we study quantum electrodynamics (or rather Maxwell's theory) in $D=(3+1)$ spacetime dimensions, and YM theory in both $D=(1+1)$ and $D=(3+1)$. In the latter case, only an approximate solution can be found and I briefly discuss possible ways to improve our findings.

\section{The Wilson loop in the Hamiltonian approach}

We study $S U(N)$ YM theory in euclidean space using the Hamiltonian approach in Weyl gauge, $A_{0}=0$. The physical energy states of this formulation are stationary wave functionals $\Psi_{n}[\mathbf{A}(\mathbf{x})] \cdot e^{-i E_{n} t}$ which obey the Schrödinger equation based on the Hamiltonian

$$
H=\frac{g^{2}}{2} \int d x \Pi^{2}(\mathbf{x})+\frac{1}{2 g^{2}} \int d x \mathbf{B}^{2} .
$$

Here, $\mathbf{A}^{a}(\mathbf{x})$ is the gauge connection, $\Pi^{a}(\mathbf{x})=-i \delta / \delta \mathbf{A}^{a}(\mathbf{x})$ its conjugate momentum, $g$ the (bare) coupling constant and $\mathbf{B}^{a}=\nabla \times \mathbf{A}^{a}-\frac{1}{2} f^{a b c} \mathbf{A}^{b} \times \mathbf{A}^{c}$ the non-Abelian magnetic field.

To proceed, we must fix the residual time-independent gauge symmetry left after imposing Weyl gauge, for which we choose the Coulomb condition, $\nabla \cdot \mathbf{A}=0$. This adds gauge-fixing terms to the Hamiltonian (which we discuss below) and imposes Gauß' law as a constraint on the physical 
states: In the sector with $n$ static charges located at positions $\mathbf{x}_{1} \ldots, \mathbf{x}_{n}$, we have $n$ additional colour indices on the wave functionals and Gauß' law reads (with generators in the right representation)

$$
\hat{\Gamma}^{a}(\mathbf{z}) \Psi_{i_{1}, \ldots i_{n}}\left(\mathbf{A} ; \mathbf{x}_{1}, \ldots, \mathbf{x}_{n}\right)=-\left\{\sum_{\ell=0}^{n} \sum_{\left\{k_{\ell}\right\}} T_{i_{\ell} k_{\ell}}^{a} \delta\left(\mathbf{z}-\mathbf{x}_{\ell}\right) \prod_{m \neq \ell} \delta_{i_{m} k_{m}}\right\} \cdot \Psi_{k_{1}, \ldots, k_{n}}\left(\mathbf{A} ; \mathbf{x}_{1}, \ldots \mathbf{x}_{n}\right) .
$$

The complete physical Hilbert space thus decomposes into orthogonal charge sectors characterised by the data from the external charges.

Next we consider a rectangular temporal Wilson loop of extension $(T \times R)$. Since $A_{0}=0$, we have $W=\operatorname{tr}\left\{U[\mathbf{A}](\mathbf{R}, \mathbf{0} ; T)^{\dagger} \cdot U[\mathbf{A}](\mathbf{0}, \mathbf{0} ; 0)\right\}$, where $U[\mathbf{A}](\mathbf{y}, \mathbf{x} ; t)$ is the parallel transporter $\mathbf{x} \rightarrow \mathbf{y}$ at fixed time $t$, in the background of the gauge potential $\mathbf{A}$. Using euclidean time evolution and the abbreviation $U(\mathbf{x}) \equiv U[\mathbf{A}](\mathbf{x}, \mathbf{0} ; 0)$, we find

$$
\langle W\rangle=\operatorname{tr}\left\langle 0\left|U(\mathbf{R})^{\dagger} e^{-\left(H-E_{0}\right) T} U(\mathbf{R})\right| 0\right\rangle=\operatorname{tr}\left\langle\mathbf{R}\left|e^{-T\left(H-E_{0}\right)}\right| \mathbf{R}\right\rangle .
$$

The salient point is now that the vacuum is gauge-invariant, while the Wilson state $|\mathbf{R}\rangle=U(\mathbf{R})|0\rangle$ is in the $q \bar{q}$-sector according to eq. (2.2). Inserting a complete set of energy eigenstates from all sectors, only the $q \bar{q}$-sector will thus contribute and we have

$$
\langle W\rangle=\operatorname{tr} \sum_{n}\left\langle 0\left|U^{\dagger}(\mathbf{R})\right| n\right\rangle_{q \bar{q}} \cdot e^{-\left(H-E_{0}\right) T} \cdot{ }_{q \bar{q}}\langle n|U(\mathbf{R})| 0\rangle=\sum_{n} e^{-T\left(E_{n}^{(q \bar{q})}-E_{0}\right)}\left\|\langle\mathbf{R} \mid n\rangle_{q \bar{q}}\right\|^{2},
$$

where $\|\Omega\|^{2} \equiv \operatorname{tr}\left(\Omega^{\dagger} \Omega\right)$. In the limit of large Euclidean time extensions, we project out the Wilson potential as the difference between the ground state energies with and without static quarks, $E_{0}^{q \bar{q}}(R)-E_{0}=-\lim _{T \rightarrow \infty} T^{-1} \ln \langle W\rangle$.

The next step is to remove the Wilson line from the state $|\mathbf{R}\rangle$ by means of a unitary transformation based on the parallel transporter $U(\mathbf{R})$. Under such a transformation, the conjugate momentum (and thus the Hamiltonian eq. (2.1)) acquires an additional term, $\Pi_{i}^{a}(\mathbf{x}) \rightarrow \widetilde{\Pi}_{i}^{a}(\mathbf{x})=\Pi_{i}^{a}(\mathbf{x})+\varepsilon_{i}^{a}(\mathbf{x})$, where

$$
\varepsilon^{a}(\mathbf{x}) \equiv \int_{0}^{\mathbf{R}} d \mathbf{y} \delta(\mathbf{x}-\mathbf{y}) U(\mathbf{y})^{\dagger} i T^{a} U(\mathbf{y})
$$

As a consequence, the Wilson loop is now computable as a zero-charge vacuum expectation value,

$$
\langle W\rangle=\operatorname{tr}\left\langle 0\left|e^{-T\left(\widetilde{H}-E_{0}\right)}\right| 0\right\rangle
$$

where $\widetilde{H}$ differs from eq. (2.1) by the momentum $\operatorname{shift}^{1} \varepsilon(\mathbf{x})$.

To illustrate the problems in extracting the true potential, let us consider the contribution to the Wilson loop eq. (2.6) which is quadratic in the induced electric field $\varepsilon(\mathbf{x})$,

$$
V_{\text {ind }}(R)=\frac{g^{2}}{2} \operatorname{tr} \int d x\left[\varepsilon^{a}(\mathbf{x})\right]^{2}=\frac{g^{2}}{2} C_{2} \delta_{\perp}^{(2)}(0) \cdot|\mathbf{R}|,
$$

where $C_{2}$ is the quadratic Casimir operator for the colour group, and the (quadratically) divergent prefactor $\delta_{\perp}(0)$ is due to the infinitely thin Wilson lines. Although $V_{\text {ind }}$ is formally confining, it

\footnotetext{
${ }^{1}$ This shift complicates the treatment of $\widetilde{H}$ considerably, since $\left[\Pi^{a}(\mathbf{x}), \varepsilon^{b}(\mathbf{x})\right] \neq 0$.
} 
must be spurious because it exists even for $G=U(1)$. On the other hand, it is also not correct to simply drop $V_{\text {ind }}$, because the true potential is hidden underneath the divergence: For $G=U(1)$,

$$
V_{\text {ind }}(R)=\frac{g^{2}}{2} \operatorname{tr} \int d x\left[\varepsilon_{\perp}(\mathbf{x})\right]^{2}+\frac{g^{2}}{2} \operatorname{tr} \int d x\left[\varepsilon_{\|}(\mathbf{x})\right]^{2},
$$

where the first factor renormalizes the Wilson loop, and the second factor gives the true (Coulomb) potential, cf. section 3.1.

The unitary transformation leading to eq. (2.6) only assumes Weyl gauge and thus works with or without Coulomb gauge fixing. In cases where the exact gauge-invariant ground state is known, it is not necessary to fix the residual gauge freedom since the Wilson loop is gauge invariant. In more realistic cases, however, the gauge invariant ground state will not be known and we have to resort to approximations based on Coulomb gauge. The required gauge-fixing is virtually impossible to effect directly in eq. (2.6), except for the Abelian model. A much better strategy is to go back to eq. (2.3), perform the standard gauge fixing and resolve Gauß' law exactly, which induces in $H \rightarrow H_{\text {fix }}$ the well-known Coulomb term, ${ }^{2}$

$$
H_{C}=\frac{g^{2}}{2} \int d^{3}(x, y) \mathscr{J}^{-1}\left[\rho^{a}(\mathbf{x})+\rho_{\text {dyn }}^{a}(\mathbf{x})\right] \mathscr{J} F^{a b}(\mathbf{x}, \mathbf{y})\left[\rho^{b}(\mathbf{y})+\rho_{\text {dyn }}^{b}(\mathbf{y})\right],
$$

where $\mathscr{J}$ is the Faddeev-Popov determinant and $F^{a b}$ the the so-called Coulomb kernel. The Wilson loop now reads

$$
\langle W\rangle=\operatorname{tr}\left\langle\mathbf{R}_{\perp}\left|e^{-T\left(H_{\text {fix }}-E_{0}\right)}\right| \mathbf{R}_{\perp}\right\rangle
$$

where the new Wilson-like state $\left|\mathbf{R}_{\perp}\right\rangle=U\left[\mathbf{A}_{\perp}\right](\mathbf{R})|0\rangle$ is no longer from the $q \bar{q}$ sector, but rather has overlap with the zero-charge vacuum. Any divergences associated with the point-like cross section of of the Wilson lines in $\left|\mathbf{R}_{\perp}\right\rangle$ must therefore be unrelated to external charges: as we will see, the extra divergences renormalize the composite operator $W \rightarrow W_{R} \equiv Z_{W}(\Lambda) \cdot W$, but they do not affect the physical potential. Whether or not the state $\left|\mathbf{R}_{\perp}\right\rangle$ in eq. (2.10) is subsequently removed from the Wilson loop by a unitary transformation $U_{\perp}(\mathbf{R})$ as described earlier becomes a matter of computational convenience.

\section{Applications}

\subsection{Quantum Electrodynamics}

In the Abelian case $G=U(1)$, the exact ground state is known and we can first attempt a gauge-invariant treatment based on eq. (2.6). The induced electric field

$$
\varepsilon(\mathbf{x})=\int_{\mathbf{0}}^{\mathbf{R}} d \mathbf{y} \delta^{(3)}(\mathbf{x}-\mathbf{y})
$$

is now field-independent, so that the induced potential (2.7) could be pulled out of the expectation value $\langle W\rangle$. In view of the discussion above, it is more convenient to first split $\varepsilon=\varepsilon^{\perp}+\varepsilon^{\|}$and pull

\footnotetext{
${ }^{2}$ As a consequence of the parallel transport, the non-Abelian charges $\rho^{a}(\mathbf{x})=\delta(\mathbf{x}-\mathbf{R}) i T^{a}-\delta(\mathbf{x}) U(\mathbf{R}) i T^{a} U(\mathbf{R})^{\dagger}$ are field-dependent.
} 
out the longitudinal piece only, $\langle W\rangle=e^{-T V_{\|}(R)} \cdot\left\langle 0\left|e^{-T\left(\widetilde{H}_{\mathrm{QED}}^{\prime}-E_{0}\right)}\right| 0\right\rangle$ where

$$
\widetilde{H}_{\mathrm{QED}}^{\prime}=\frac{1}{2} \int d^{3} x\left\{g^{2}\left[\Pi^{\perp}(\mathbf{x})+\varepsilon^{\perp}(\mathbf{x})\right]^{2}+\frac{1}{g^{2}}\left[\nabla \times \mathbf{A}^{\perp}(\mathbf{x})\right]^{2}\right\} .
$$

It is now convenient to reverse the unitary transformation that led to eq. (2.7), but this time with the transversal gauge connection $\mathbf{A}^{\perp}$ only. As a result, the shift in the electric field is removed from the Hamiltonian (3.2) and reshuffled into the state $\left|\mathbf{R}^{\perp}\right\rangle=U\left[\mathbf{A}^{\perp}\right](\mathbf{R})|0\rangle \equiv U^{\perp}(\mathbf{R})|0\rangle$. Although this new state resembles the initial Wilson state, it is gauge-invariant as it depends on $\mathbf{A}^{\perp}$ only. Inserting a complete set of eigenstates of the standard QED Hamiltonian eq. (3.2) (without $\varepsilon^{\perp}$ ) yields

$$
\langle W\rangle=e^{-T V_{\|}(R)} \cdot \sum_{n}\left|\left\langle 0\left|U^{\perp}(\mathbf{R})\right| n\right\rangle\right|^{2} e^{-T\left(E_{n}-E_{0}\right)} \stackrel{T \rightarrow \infty}{\longrightarrow}\left|\left\langle 0\left|U^{\perp}(\mathbf{R})\right| 0\right\rangle\right|^{2} \cdot e^{-T V_{\|}(R)},
$$

provided that $\left|\mathbf{R}^{\perp}\right\rangle$ has non-vanishing overlap with the true vacuum. For Maxwell's theory the ground state is Gaussian and the relevant matrix element can be computed exactly. Using a $O(3)$ invariant UV cutoff $\Lambda$ for the momentum integration, we find that $Z_{W}$ formally vanishes in the limit $\Lambda \rightarrow \infty$ because $\left|\mathbf{R}^{\perp}\right\rangle$ has poor overlap with the true ground state [9]. On the other hand, $Z_{W}$ is also independent of the temporal extension $T$ of the loop and hence cannot contribute to the physical potential.

The correct interpretation is given by the operator product expansion (OPE): Since the loop operator $W$ contains products of field operators at arbitrarily close points, we expect short distance $(\Lambda \rightarrow \infty)$ divergences associated with $W$, on top of the counter terms necessary to render Green functions finite. In fact, it has been known for a long time [11, 12] that one overall multiplication $W \rightarrow W_{R} \equiv Z_{W}^{-1} \cdot W$ is sufficient to render $\left\langle W_{R}\right\rangle=e^{-T V_{\perp}}$ finite. From eq. (3.1), the true physical potential becomes

$$
V(R)=V_{\|}(R)=\frac{g^{2}}{2} \int d x\left[\varepsilon^{\|}(\mathbf{x})\right]^{2}=\frac{g^{2}}{4 \pi R}-\frac{g^{2}}{4 \pi|\mathbf{0}|} .
$$

This is just the usual Coulomb potential including the self-energy of the charges.

The entire derivation could also be repeated in a Coulomb gauge fixed formulation. If we start from eq. (2.6), the gauge fixing gives $\widetilde{H} \rightarrow \widetilde{H}_{\text {fix }}=H_{\text {QED }}^{\prime}+V_{\|}$, cf. eq. (3.2), and the computation becomes identical to the gauge-invariant treatment above. Alternatively, we could also start from eq. (2.3) and resolve Gauß' law for the state $|\mathbf{R}\rangle$ explicitly, which induces the Coulomb term $H \rightarrow$ $H_{\text {fix }}=H_{\mathrm{QED}}\left[\mathbf{A}_{\perp}\right]+H_{C}$ in the Hamiltonian. This time, the Coulomb potential comes directly from $H_{C}$, while the first term in $H_{\text {fix }}$ only renormalizes the Wilson loop.

\subsection{Yang-Mills Theory in $D=1+1$}

This model is (almost) topological and requires a non-contractible spactime manifold to give nontrivial results. Since time must be unrestricted in the Hamiltonian formalism, we compactify space to an interval $[0, L]$ with periodic boundary conditions and take spacetime to be the cylinder $\mathrm{M}=\mathbb{R} \times S^{1}$. For this model, the induced potential eq. (2.7) gives the correct string tension

$$
\sigma_{1+1}=\frac{g^{2}}{2} C_{2} \stackrel{S U(2)}{=} \frac{3}{8} g^{2}
$$


In view of the above discussion, it is, however, unclear why this should be the correct answer, in particular since $V_{\text {ind }}=\sigma_{1+1} R$ is not periodic or invariant under $R \rightarrow L-R$ as expected from the compactification.

To address this issue, we go back to eq. (2.3) and fix the minimal Coulomb gauge $\partial_{1} A_{1}^{a}=0$ and $A_{1}^{a}=$ diag. Taking the colour group $G=S U(2)$ for simplicity, we are thus left with only one scalar degree of freedom

$$
\vartheta \equiv \frac{1}{2} A_{1}^{3} L \in[0, \pi]
$$

The restriction to the compact interval (the fundamental modular region in this case) eliminates all residual gauge symmetries. In order to fix eq. (2.3), we have to determine the gauge fixed Hamiltonian in the $q \bar{q}$-sector to which the Wilson state $|R\rangle$ belongs. This has two pieces

$$
H_{\mathrm{fix}}=H_{\mathrm{fix}}^{0}+H_{C}=H_{\mathrm{fix}}+\frac{g^{2}}{2} \int d(x, y) \rho^{a}(x) F^{a b}(x, y) \rho^{b}(y),
$$

where the non-Abelian charge interaction comes from the resolution of Gauß' law. The zero-charge Hamiltonian $H_{\text {fix }}^{0}$ and its spectrum have been studied thoroughly in ref. [13]. As for the Coulomb term, we have to take into account that the external charges $\rho^{a}$ are field-dependent due to the parallel transport. Expanding all colour vectors in a polar basis $\left(\mathbf{e}_{0}=e_{3}, \mathbf{e}_{ \pm}=\left(\mathbf{e}_{1} \pm \mathbf{e}_{2}\right) / \sqrt{2}\right)$, we find

$$
H_{C}=\frac{g^{2}}{8} \sum_{\sigma=-1}^{1} \tau_{\sigma} \tau_{\sigma}^{\dagger}\left\{2 F_{\sigma}(0 ; \vartheta)-\left[e^{-2 i \sigma(R / L) \vartheta} F_{\sigma}(R ; \vartheta)+\mathrm{cc}\right]\right\},
$$

where $\tau_{\sigma}$ are the polar Pauli matrices. The Coulomb kernel $F_{\sigma}(R ; \vartheta)$ in the polar basis has also been determined in ref. [13] by a Fourier expansion which is strictly periodic in $R$. In the first period $|R|<L$, the series can actually be resummed to give the simple result

$$
H_{C}=\frac{g^{2}}{8}\left[3|R|-\frac{R^{2}}{L}\right] \mathbb{1} \equiv V_{C}(R) \mathbb{1} ;
$$

for $|R|>L$ it must be continued periodically. Quite surprisingly, this turns out to be independent of the gauge field $\vartheta$ and can thus be pulled out of the gauge-fixed expectation value eq. (2.3). Inserting again the complete set of eigenstates $\langle\vartheta \mid n\rangle=\Psi_{n}(\vartheta)$ of $H_{\text {fix }}^{0}$, we arrive at

$$
\langle W\rangle=\operatorname{tr}\left\langle R\left|e^{-T\left(H_{\mathrm{fix}}+H_{C}-E_{0}\right)}\right| R\right\rangle \stackrel{T \rightarrow \infty}{\longrightarrow} \operatorname{tr}|\langle 0|U(R)| 0\rangle|^{2} \cdot e^{-T V_{C}(R)} .
$$

The prefactor $Z_{W}=\operatorname{tr}|\langle 0|U(R)| 0\rangle|^{2}$ can be computed explicitly and turns out to $T$-independent (and finite). Again, $Z_{W}$ is the OPE renormalization constant for the composite operator $W$ and we obtain the renormalized Wilson loop $\left\langle W_{R}\right\rangle=e^{-T V_{C}(R)}$. Since $V_{C}(R)$ is periodically continued for $|R|>L$, we have to go to the limit $L \gg R$ to avoid finite size effects, and the effective string tension becomes

$$
\sigma_{1+1}=\left.\frac{d V_{C}(R)}{d R}\right|_{R=0}=\frac{3}{8} g^{2} .
$$

This agrees with eq. (3.5) and is also the known expression from the literature. It remains to explain why the physical potential $V_{C}(R)$ is periodic in $R$, but not invariant under $R \rightarrow L-R$ as it should 
be on a compactified $[0, L] \simeq S^{1}$. A careful analysis [10] along the lines in sec. 2 reveals that the physical potential between static quarks is not given by any single Wilson loop once the space direction is compactified. Instead, all the equivalent loops with $R \rightarrow R+m L(m \in \mathbb{Z})$ compete and the one giving the minimal potential dominates in the limit $T \rightarrow \infty$. Taking this prescription into account, the level crossing between $|R\rangle$ and $|R-L\rangle$ at $R=L / 2$ yields a potential $V(R)$ that is both invariant and symmetric about $R=L / 2$.

\subsection{Yang-Mils Theory in $D=3+1$}

For the physically most intersting case of YM theory in $D=3+1$, we start again from eq. (2.3) and introduce the usual Coulomb gauge fixing in the Hamiltonian, combined with the resolution of Gauß' law in the $q \bar{q}$ sector to which the Wilson state $|\mathbf{R}\rangle$ belongs,

$$
\langle W\rangle=\operatorname{tr}\left\langle\mathbf{R}_{\perp}\left|e^{-T\left(H_{\mathrm{YM}}^{\mathrm{fix}}+H_{C}\left[\rho_{\mathrm{dyn}}+\rho\right]-E_{0}\right)}\right| \mathbf{R}_{\perp}\right\rangle
$$

In view of the approximations below, it is expedient to perform the unitary transformation from section 2 and reshuffle the parallel transporter $U\left[\mathbf{A}^{\perp}\right](\mathbf{R})$ from the Wilson state into the Hamiltonian. As a result, we have

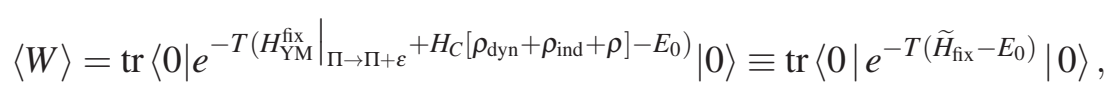

where the momentum $\Pi$ in the gauge-fixed vacuum Hamiltonian is shifted by the induced electric field eq. (2.5), and the Coulomb term eq. (2.9) will now contain an induced charge $\rho_{\text {ind }}=$ $\left[-\mathbf{A}^{\perp}, \varepsilon^{\perp}\right]$, in addition to the dynamic charge $\rho_{\mathrm{dyn}}=\left[-\mathbf{A}^{\perp}, \Pi^{\perp}\right]$ of the gluon and the external charge $\rho$ of the static quarks. To proceed, we decompose the complete Hamiltonian $\widetilde{H}_{\text {fix }}$ from eq. (3.13) into three pieces, $\widetilde{H}_{\text {fix }}=H_{\text {fix }}^{0}+\widetilde{V}_{C}+\Delta H$, where $H_{\text {fix }}^{0}$ is the usual Coulomb Hamiltonian in the absence of external charges, $\widetilde{V}_{C}$ is the non-Abelian Coulomb interaction between the external charges, and $\Delta H$ containing the remaining (indirect) charge interactions.

In order to evaluate eq. (3.13) with this decomposition, we have resort to a sequence of approximations, which are not all under good control:

1. Jensen's inequality: $\langle W\rangle \geq \operatorname{tr} \exp \left[-T\left\langle 0\left|\widetilde{H}^{\mathrm{fix}}-E_{0}\right| 0\right\rangle\right]=\operatorname{tr} \exp \left[-T\left\langle 0\left|\widetilde{V}_{C}+\Delta H\right| 0\right\rangle\right]$

2. Abelization: $\quad\left[U^{\perp}(R), T^{a}\right] \approx 0$

3. Factorization: $\quad\left\langle 0\left|Q^{a} F^{a b} Q^{\prime b}\right| 0\right\rangle \approx\left\langle 0\left|Q^{a} Q^{\prime b}\right| 0\right\rangle\left\langle 0\left|F^{a b}\right| 0\right\rangle \equiv\left\langle 0\left|Q^{a} Q^{\prime a}\right| 0\right\rangle \bar{F}$

Approximation (1.) allows to drop $H_{\text {fix }}^{0}$, while (2.) removes terms involving the conjugate momentum $\Pi$ and $\varepsilon^{\perp}$, and (3.) simplifies the remaining pieces in $\Delta H$ and, in particular, the Coulomb interaction, $\widetilde{V}_{C}$. After a lengthy calculation [10], the Wilson loop is thus reduced to a form which requires, as only input from the ground state, the gluon propagator $\left\langle 0\left|A_{i}^{a}(\mathbf{x}) A_{j}^{b}(\mathbf{y})\right| 0\right\rangle=$ $\delta^{a b} t_{i j} D(\mathbf{x}-\mathbf{y})$ and the vev. of the Coulomb kernel, $\left\langle 0\left|F^{a b}(\mathbf{x}-\mathbf{y})\right| 0\right\rangle=\delta^{a b} \bar{F}(\mathbf{x}-\mathbf{y})$ :

$$
\begin{aligned}
\langle W\rangle \approx & \exp \left\{-T \frac{g^{2}}{2} N_{C} \int d^{3}(x, y) \varepsilon_{a}^{\perp}(\mathbf{x})\left[N_{C}^{-1} \delta(\mathbf{x}-\mathbf{y})+\bar{F}(\mathbf{x}-\mathbf{y}) D(\mathbf{x}-\mathbf{y})\right] \varepsilon_{a}^{\perp}(\mathbf{y})\right\} \times \\
& \times \exp \left\{-T C_{2} g^{2}[\bar{F}(R)-\bar{F}(0)]\right\} .
\end{aligned}
$$


The first term in the exponent is again the self-energy of the Wilson lines which gives rise to a renormalization $Z_{W}$ of the loop operator. The large distance behaviour of the second term is dominated by the small momentum form of the correlators, for which we use the relations $D(k) \sim\left[k^{2}+M^{4} / k^{2}\right]^{-\frac{1}{2}}$ and $\bar{F}(k) \sim k^{-4}$ suggested by both variational calculations [4] and recent lattice simulations [7]. It can then be shown that the second piece in the exponent of eq. (3.14) is subdominant $\left(\sim R^{-1}\right)$ at large distances. The dominant contribution to the Wilson potential is given, in our approximation, by the explicit Coulomb interaction, and the Wilson string tension equals the Coulomb string tension, $\sigma_{W} \approx \sigma_{C}$.

\section{Conclusions}

In this talk, I have presented a recent calculation of the Wilson potential in the Hamiltonian approach to Yang-Mills theory. A careful analysis of Gauß' law and the physical degrees of freedom combined with unitary transformations to take care of time evolution reveal that the spurious divergences in the Wilson potential can be absorbed in OPE renormalizations of the composite loop operator. Using this formalism, the correct result could be derived in cases where the exact ground state is known. For the physically most interesting case of Yang-Mills theory in $D=(3+1)$, only an approximate solution could be obtained in which the Wilson and Coulomb string tension equal (while $\sigma_{W} / \sigma_{C} \simeq 0.3 \ldots 0.5$ in lattice simulations). The main reason for this discrepancy is most likely the approximate factorization used in the calculation, which neglects the screening of external charges by gluons. One-loop calculations of this contribution indeed indicate a reduction of $\sigma_{W}$, and further investigations of this issue are in preparation.

\section{References}

[1] V. Gribov. Nucl.Phys. B139, 1 (1978).

[2] D. Zwanziger. Nucl.Phys. B485, 185 (1997).

[3] A. P. Szczepaniak and E. S. Swanson. Phys.Rev. D65, 025012 (2002).

[4] D. Epple, H. Reinhardt, and W. Schleifenbaum. Phys.Rev. D75, 045011 (2007).

[5] H. Reinhardt. Phys.Rev.Lett. 101, 061602 (2008).

[6] G. Burgio, M. Quandt, and H. Reinhardt. Phys.Rev.Lett. 102, 032002 (2009).

[7] G. Burgio, M. Quandt, and H. Reinhardt. Phys.Rev. D86, 045029 (2012).

[8] D. Zwanziger. Phys.Rev.Lett. 90, 102001 (2003).

[9] P. E. Haagensen and K. Johnson. [hep-th/9702204] (1997).

[10] H. Reinhardt, M. Quandt, and G. Burgio. Phys.Rev. D85, 025001 (2012).

[11] J.-L. Gervais and A. Neveu. Nucl.Phys. B163, 189 (1980).

[12] A. M. Polyakov. Nucl.Phys. B164, 171 (1980).

[13] H. Reinhardt and W. Schleifenbaum. Annals Phys. 324, 735 (2009). 